\title{
Dental Sleep Medicine redefined
}

\author{
Ghizlane Aarab ${ }^{1} \cdot$ Frank Lobbezoo $^{1}$ (D)
}

Received: 26 June 2018 / Revised: 30 June 2018 / Accepted: 12 July 2018 / Published online: 19 July 2018

(C) Springer Nature Switzerland AG 2018

Dear Editor,

In 1999, Prof. Gilles Lavigne (Université de Montréal, QC, Canada) and coworkers published a comprehensive review, entitled 'Sleep disorders and the dental patient' [1]. This landmark article was the first to describe in detail several common sleep disorders of interest to dentists, viz., not only snoring and obstructive sleep apnea but also sleep bruxism, xerostomia, hypersalivation, gastroesophageal reflux, and orofacial pain. Over the years, the publication by Lavigne et al. [1] was cited mainly in dental journals, which suggests good visibility of the topic amongst dental researchers.

Consequently, one would have expected a broadening of Dental Sleep Medicine as a discipline within dentistry. However, Dental Sleep Medicine Academies worldwide kept their focus on the management of sleep-related breathing disorders, including snoring and obstructive sleep apnea (OSA), with oral appliance therapy and upper airway surgery. Since sleep disorders other than sleep-disordered breathing may have oral and maxillofacial causes and consequences, Lobbezoo et al. [2] formulated a new definition for Dental Sleep Medicine:

'Dental Sleep Medicine is the discipline concerned with the study of the oral and maxillofacial causes and consequences of sleep-related problems.'

This new definition broadens the discipline to the diagnosis and management of other conditions where den-

Ghizlane Aarab

g.aarab@acta.nl

1 Department of Oral Health Sciences, Academic Centre for Dentistry Amsterdam (ACTA), University of Amsterdam and Vrije Universiteit Amsterdam, Gustav Mahlerlaan 3004, 1081

LA Amsterdam, The Netherlands tistry plays a pivotal role, viz., orofacial pain, oral moistening disorders (oral dryness, hypersalivation), gastroesophageal reflux disorder, sleep-related breathing disorders (snoring, obstructive sleep apnea), and mandibular movement disorders (dyskinesia, dystonia, sleep bruxism). Apart from the inclusion of more sleep-related problems than only sleep-disordered breathing in the discipline of Dental Sleep Medicine, the new definition also does justice to the increasing insight that the above-mentioned conditions are associated with each other. For example, evidence is growing that obstructive sleep apnea events are frequently followed by sleep bruxism events, which suggests that sleep bruxism helps to restore the patency of the upper airway in obstructive sleep apnea patients [3]. This means that an isolated focus on sleep-related breathing disorders might lead to a tunnel vision, while a broader knowledge of the whole range of dental sleep disorders and their associations is imperative in the interest of providing the best possible management for every single one of our patients.

Based on the above, we express our sincere hope that the readership of Sleep and Breathing will adopt the new definition of Dental Sleep Medicine in their clinics or when performing research projects within this intriguing discipline.

\section{References}

1. Lavigne GJ, Goulet JP, Zuconni M, Morisson F, Lobbezoo F (1999) Sleep disorders and the dental patient: a review of diagnosis, pathophysiology and management. Oral Surg Oral Med Oral Pathol Oral Radiol Endod 88:257-272

2. Lobbezoo F, Aarab G, Wetselaar P, Hoekema A, de Lange J, de Vries N (2016) A new definition of Dental Sleep Medicine. J Oral Rehabil 43:786-790

3. Manfredini D, Guarda-Nardini L, Marchese-Ragona R, Lobbezoo F (2015) Theories on possible temporal relationships between sleep bruxism and obstructive sleep apnea events. An expert opinion. Sleep Breath 19:1459-1465 